\title{
Low kV Analysis and First Principles Study of the Structure and Bonding at $\mathrm{SrTiO}_{3} / \mathrm{GaAs}$ Hetero-interfaces
}

\author{
Q. Qiao, R.F. Klie, S. Ogut
}

Department of Physics, University of Illinois at Chicago, Chicago, IL 60607

Ultrathin metal-oxide films on polar semiconductor surfaces have received much attention in recent years due to the occurrence of novel interfacial properties, including ferroelectricity, superconductivity and the presence of an interfacial 2-dimensional electron gas [1]. In this study, we examine the atomic and electronic structures of epitaxial ultrathin $\mathrm{SrTiO}_{3}$ (100) films on $\mathrm{GaAs}(001)$ using aberration corrected atomic-resolution Z-contrast imaging and electron energy loss spectroscopy (EELS) in combination with first principles calculations to develop a fundamental understanding of the interfacial structure-property relationships.

Initially, two different kinds of ultra-thin $\mathrm{SrTiO}_{3}$ films were deposited on As-terminated GaAs (100), one with a Ti pre-layer and the other with the $\mathrm{SrTiO}_{3}$ in direct contact with the GaAs support. So far, a detailed atomic-resolution study of this interface was not possible due to the high sensitivity of the interface to the electron beam. Recent changes in instrumentation design now allow for atomic resolution Z-contrast imaging, as well as EELS at electron energies as low as $80 \mathrm{kV}$. Figure 1 shows high-resolution Z-contrast images of the two different $\mathrm{SrTiO}_{3}$ films using the aberration-corrected JEOL ARM200-CF operated at $80 \mathrm{kV}$. No sign of beam damage has been detected even after repeated exposure. It is interesting to note here that the interface appears abrupt and no surface reconstruction of the GaAs (001) surface is observed. Using atomic-column resolved EELS, we will examine the stoichiometry of the $\mathrm{SrTiO}_{3}$ films, and show that Ti diffuses into the first few monolayers of GaAs depending on the thin film growth condition (Figure 1c and 1d).

The effects of Ti diffusion into subsurface GaAs $(001)$ with $(4 \times 2)-\beta 2$ surface reconstructions are investigated via first principles calculations. The $(4 \times 2)-\beta 2$ surface is divided into a $10 \times 5$ uniform grid. The Ti atom placed at each grid point is allowed to relax only in the direction perpendicular to the surface, along with the Ga and As atoms in the top two layers. The results are then interpolated to a finer $50 \times 50$ grid. The Ti diffusion into the subsurface GaAs (001) with $(4 \times 2)-\beta 2$ surface reconstructions is evident, as shown in the binding energy profile, Figure 2. We will also discuss the results for the formation energies of Ti-related impurity defects in the bulk and surface regions of $\mathrm{GaAs}$ to help in the interpretation of electron microscopy experiments [2].

In this presentation, we will utilize low $\mathrm{kV}$ Z-contrast imaging and EELS with atomic resolution in combination with first-principles calculations to show the effects of Ti diffusion into subsurface GaAs (001) in stabilizing the semiconducting interface [3].

\section{References}

[1] J.W. Reiner et al., Adv. Mater. 22 (2010) 2919.

[2] C.G. Van de Waal, J. Neugebauer, J. Appl. Phys. 95 (2004) 3851.

[3] This research was supported by the National Science Foundation under Grant No. DMR0604964, and also supported by the NSF CAREER Award DMR-0846748. 

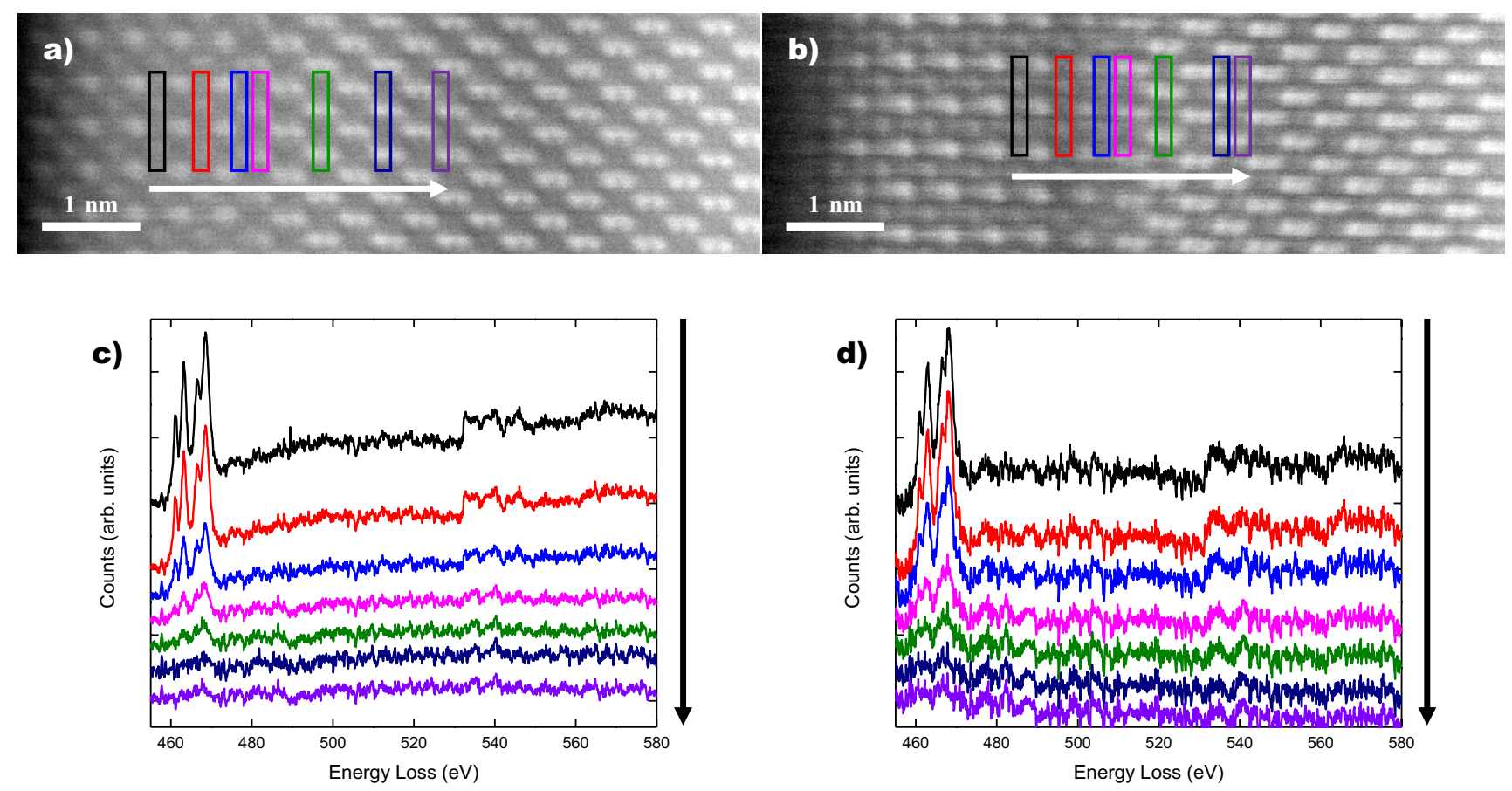

FIG. 1. Z-contrast images and EELS spectra of the $\mathrm{SrTiO}_{3}$ film on GaAs for two different growth conditions. The $\mathrm{SrTiO}_{3}$ is shown in the [001] orientation while the GaAs is seen along the [110] in both samples. a): $\mathrm{SrTiO}_{3}$ film deposited on $\mathrm{GaAs}$ after depositing a Ti pre-layer; b): same growth conditions for $\mathrm{SrTiO}_{3}$ except that no Ti pre-layer was deposited; c) and d): Ti L and O K edges extracted from the area scan, the rectangles in a) and b) highlight the individual scan areas respectively.

a)

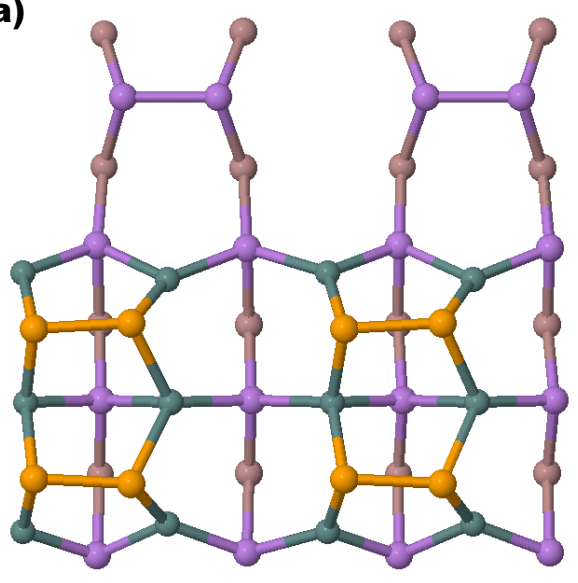

b)

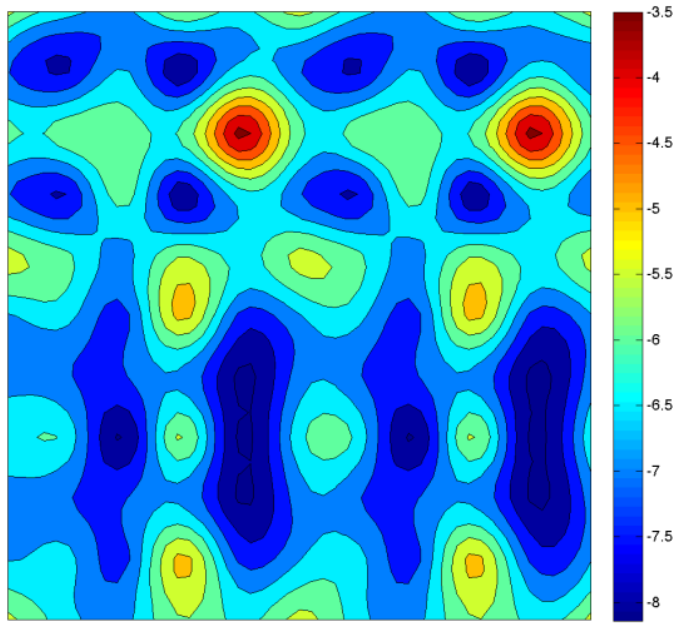

FIG. 2. Binding energy profile for Ti on the GaAs $(001)$ with $(4 \times 2)-\beta 2$ surface reconstructions. a): ball and stick model of the GaAs (001) with $(4 \times 2)-\beta 2$ surface reconstructions, a $4 \times 4$ surface cell is shown; b): the binding energy contour map of the GaAs (001) with $(4 \times 2)-\beta 2$ surface reconstructions in a), red end indicates minimum binding energy, blue end indicates maximum binding energy. 